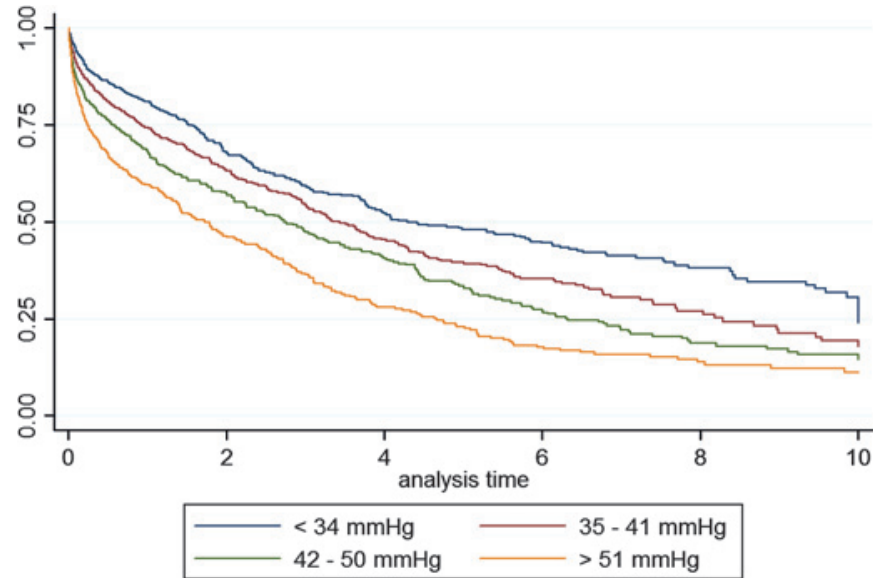

Abstract 101 Figure 1 Survival of RVSP quartile.

Conclusion An RVSP of greater than $42 \mathrm{~mm} \mathrm{Hg}$ is predictive of increased mortality in heart failure. This is finding is independent of LVSD and COPD.

\section{ETHNIC DIFFERENCES IN ENDOTHELIAL FUNCTION IN CHRONIC HEART FAILURE}

doi:10.1136/heartjnl-2011-300198.102

${ }^{1} \mathrm{E}$ Shantsila, ${ }^{2} \mathrm{P}$ S Gill, ${ }^{3} \mathrm{G}$ Y H Lip. ${ }^{1}$ University of Birmingham Centre for Cardiovascular Sciences, City Hospital, Birmingham, UK; ${ }^{2}$ University of Birmingham, Primary Care and Populational Sciences, Birmingham, UK; ${ }^{3}$ University of Birmingham Centre for Cardiovascular Science, Birmingham, UK

Background Endothelial dysfunction is characteristic of patients with heart failure (HF) and is associated with an increased risk of future cardiovascular events. However, data on ethnic differences in endothelial function in HF are scarce. In this study we aimed to compare parameters of macro- and micro-vascular endothelial function and arterial elasticity in HF age- and sex-matched patients of different ethnic origin: (i) white European, (ii) south Asian and (iii) African-Caribbean. Additionally, SA patients with systolic HF were compared to two matched control groups: (i) south Asian patients with coronary artery disease without HF(disease controls) and (ii) south Asian "healthy controls".

Methods We recruited 186 age/sex-matched patients with HF (ejection fraction $<40 \%$ ) of SA ( $n=43$, age $66.5 \pm 11.1$ years), white $(n=44$, age $68.4 \pm 9.4$ years) and African-Caribbean ( $n=21$, age 69.2 \pm 10.3 years)

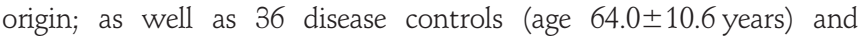
40 healthy controls ( $n=40$, age $63.3 \pm 9.24$ years). Macrovascular endothelial function was assessed as brachial artery flow mediated dilation in response to hyperaemia (FMD) and glyceryltrinitrate were assessed by vascular ultrasonography (iE33, Philips, USA). Microvascular endothelial function was evaluated by laser Doppler flowmetry of forearm skin (DRT4, Moor Instruments, UK) after iontophoresis of acetylcholine and sodium nitroprusside. Arterial stiffness was quantified by pulse wave velocity and augmentation index using (Sphygmocor, Australia).

Results Compared to disease controls and healthy controls south Asian patients with HF had impaired microvascular response to acetylcholine $(390 \pm 302 \%, 549 \pm 264 \%$, and $123 \pm 95.5 \%$, respectively, $\mathrm{p}<0.05)$ and reduced FMD $(7.12 \pm 3.64 \%, 11.8 \pm 4.66 \%$, and $4.86 \pm 4.88 \%$, respectively). HF patients of south Asian origin had impaired microvascular endothelial function (response to acetylcholine123 $\pm 95.5 \%$ ) compared to white $(258 \pm 15.6 \%)$ and African-Caribbean $(286 \pm 17.3 \%)$ groups $(p>0.05)$. HF patients of white origin had higher FMD than south Asian (4.86 $\pm 4.88 \%)$ and African-Caribbean $(5.36 \pm 3.24 \%)$ patients $(p<0.05)$. No difference in glyceryltrinitrate- and sodium nitroprusside-mediated (endothelialindependent) response was observed between study groups. In south Asian subjects, parameters of pulse wave velocity and augmentation index did not differ between those with HF and those in control groups. No ethnic differences were detected in pulse wave velocity. Conclusion: South Asian patients with HF have impaired micro- and macro-vascular endothelial function, but preserved arterial elastic properties. Significant ethnic differences in endothelial function are present in patients with HF.

\section{SENILE SYSTEMIC AMYLOIDOSIS: A COMMON CAUSE OF HEART FAILURE IN THE ELDERLY?}

doi:10.1136/heartjnl-2011-300198.103

${ }^{1} \mathrm{~J} \mathrm{H}$ Pinney, ${ }^{2} \mathrm{H} \mathrm{J}$ Lachmann, ${ }^{2} \mathrm{~J} \mathrm{D}$ Gillmore, ${ }^{2} \mathrm{~A}$ Wechalekar, ${ }^{3} \mathrm{~S} \mathrm{D} \mathrm{J}$ Gibbs, ${ }^{3} \mathrm{P}$ Sattianayagam, ${ }^{4,5} \mathrm{~S} \mathrm{M}$ Banypersad, ${ }^{6,7} \mathrm{~J}$ Dungu, ${ }^{3} \mathrm{~N}$ Wassef, ${ }^{3} \mathrm{C}$ A McCarthy, ${ }^{3} \mathrm{P}$ N Hawkins, ${ }^{3} \mathrm{C}$ J Whelan. ${ }^{1}$ National Amyloidosis Centre and UCL Centre for Nephrology, UCL Division of Medicine, Royal Free Hospital, London, UK; ${ }^{2}$ National Amyloidosis Centre, UCL Division of Medicine, Royal Free Hospital, London, UK ${ }^{3}$ National Amyloidosis Centre, UCL Medical School, Royal Free Hospital, London, UK; ${ }^{4}$ National Amyloidosis Centre, London, UK; ${ }^{5}$ The Heart Hospital, UCL Medical School, London, UK; ${ }^{6}$ National Amyloidosis Centre, UCL Medical School, University of London, London, UK; ${ }^{7}$ St George's Hospital, University of London, London, UK

Senile systemic amyloidosis (SSA) is a rare cause of heart failure due to the deposition of wildtype transthyretin. The clinical features and outcome are ill defined; our aim was to evaluate the natural history of the disease in the UK in a group of thoroughly characterised patients. The series included all cases of biopsy proven transthyretin (TTR) amyloidosis with wildtype TTR gene sequencing who were prospectively followed up between January 2001 and May 2010. Clinical, biochemical, ECG and echocardiographic evaluation were performed at presentation to our centre. Patient survival was estimated using Kaplan-Meier analysis. 55 patients with histologically proven SSA; 36 (65.5\%) from cardiac, 14 (25.4\%) from GI tract, 3 (5.5\%) from bladder, 1 (1.8\%) from fat and $1(1.8 \%)$ from carpal tunnel tissue were identified. 49 (89\%) were male. The median age at diagnosis and death were 74 (range 66-89) and 79 (range 69-84) years respectively. Survival from symptom onset and diagnosis was 7.04 (range 0.54-8.41) and 4.58 (range $0.07-5.41$ ) years respectively. In recent years more patients have been diagnosed with 2 (3.6\%), $14(25.5 \%)$ and $39(70.9 \%)$ patients between 2001-2003, 2004-2006 and 2007-2009 respectively. The most common presentation was with breathlessness in 28 patients $(51 \%)$. Twenty-four patients $(43.6 \%)$ had prior carpal tunnel operations. Twelve $(21.8 \%)$ patients had a history of ischaemic heart disease. Fifteen had had a coronary angiogram; 8 were reportedly normal and 7 required intervention. Arrhythmias were common, 20 patients (36.3\%) had a history of atrial fibrillation and $6(10.9)$ had pacemakers in situ. ECG findings were; 24 (43.6\%) in AF, $6(10.9 \%)$ first degree block, 10 (18.2\%) left bundle and $6(10.9 \%)$ right bundle branch block, 27 (49\%) Twave changes, $11(20 \%)<5 \mathrm{~mm}$ complexes in all inferior leads. Echocardiographic findings revealed the median IVSd was 1.7 (range 1.1-2.5) cm, median E/A ratio was 2.7 (range 0.79-5.4), E/E' 15.81 (range 7.5-41.1) and ejection fraction was 45.5 (range 13-83)\%. Blood results showed; the median baseline NT-proBNP was 356.1 (range 5-2611) and troponin T 0.03 (range $0.01-0.28)$. Twenty-five patients had a troponin $\mathrm{T}>0.03(45 \%)$. Ten patients $(18 \%)$ had a detectable paraprotein and $2(3.6 \%)$ had bence jones proteins. SSA is present in $>25 \%$ of the very elderly at post mortem but was rarely diagnosed during life. It is becoming more frequently recognised perhaps due to widespread use of cardiac MRI. Most patients are male but women can be affected. A history of carpal tunnel syndrome is common. The diagnosis is often made after the onset of breathlessness. Systolic and diastolic dysfunction 
can be seen on echocardiogram. A positive troponin is a common finding with a subsequent normal coronary angiogram. Incidental paraproteins are prevalent in up to $8 \%$ of this population and it is important to obtain a tissue diagnosis to rule out AL amyloidosis. With supportive management medium term outcomes are good.

\section{PROGNOSTIC UTILITY OF CALCULATED PLASMA VOLUME STATUS IN CHRONIC HEART FAILURE}

doi:10.1136/heartjnl-2011-300198.104

${ }^{1} \mathrm{H}$ Z Ling, ${ }^{1} \mathrm{~N}$ Aung, ${ }^{1,2} \mathrm{~J}$ Flint, ${ }^{1,2} \mathrm{~S}$ Aggarwal, ${ }^{1,2} \mathrm{~S}$ Weissert, ${ }^{1,2} \mathrm{~A}$ Cheng, ${ }^{3} \mathrm{D}$ P Francis, ${ }^{3} \mathrm{~J}$ Mayet, ${ }^{1,2} \mathrm{M}$ Thomas, ${ }^{1,2} \mathrm{~S}$ Woldman, ${ }^{1,2,4} \mathrm{D} 0$ Okonko. ${ }^{1}$ University College London Hospital, London, UK; ${ }^{2}$ The Heart Hospital, London, UK; ${ }^{3}$ International Center for Circulatory Health, NHLI, Imperial College London, London, UK; ${ }^{4} \mathrm{NHLI}$ Imperial College London, London, UK

Background Plasma volume (PV) expansion is a hallmark feature of worsening heart failure that is notoriously underestimated by clinical examination. While radioisotope assays optimally quantify PV status, numerous haemodialysis-based equations also exist for its estimation. The prognostic utility of such formulas in chronic heart failure (CHF) is unknown.

Methods We analysed the relation between estimated PV status and mortality in 246 outpatients with CHF (mean $( \pm S D)$ age $67 \pm 13$ years, NYHA class $2 \pm 1$, LVEF $28 \pm 8 \%$ ). PV status was calculated (Hakim RM, et al) by subtracting the patients actual PV ((1-haematocrit) $\times$ $(a+(b \times$ weight $)) ; a$ and $b$ are gender-specific constants $)$ from their ideal PV ((c $\times$ weight); $c=$ gender-specific constant).

Results Median ( \pm IOR) PV status was-261 $\pm 550 \mathrm{ml}$ with $78 \%$ and $21 \%$ of patients having PV contraction and expansion, respectively. Patients with PV excess had significantly higher creatinine and lower albumin levels. Over a median follow-up of $13 \pm 16$ months, $36(15 \%)$ patients died. PV status predicted mortality (HR 1.001, 95\% CI 1.001 to $1.002, p=0.001$ ) in a graded fashion (Abstract 104 figure $1 \mathrm{~A}$ ) and did so independently of NYHA class, LVEF, weight, haematocrit and creatinine. A PV status $\leq-178 \mathrm{ml}$ optimally predicted survival (ROC AUC $0.68, p=0.0007$ ) and conferred a $75 \%$ reduced hazard for death (HR 0.16, 95\% CI 0.07 to 0.37, p<0.0001, Abstract 104 figure 1B).
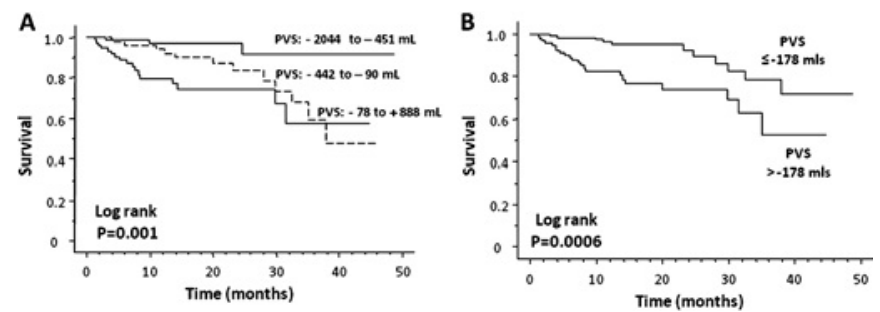

Abstract 104 Figure 1

Conclusions Calculating plasma volume status in CHF patients appears prognostically useful and suggests that dehydration is better tolerated than volume excess in these individuals and that targeting therapy to achieve a plasma volume status $\leq 178 \mathrm{ml}$ might increment survival.

\section{CLINICAL AND ECHOCARDIOGRAPHIC DETERMINANTS OF N-TERMINAL PRO B-TYPE NATRIURETIC PEPTIDE LEVEL IN PATIENTS WITH STABLE CHRONIC OBSTRUCTIVE AIRWAYS DISEASE: A PROSPECTIVE OBSERVATIONAL STUDY OF 140 PATIENTS}

doi:10.1136/heartjnl-2011-300198.105

${ }^{1} \mathrm{C}$ P Gale, ${ }^{2} \mathrm{~J}$ White, ${ }^{2} \mathrm{~A}$ Hunter, ${ }^{3} \mathrm{~J}$ Owen, ${ }^{4} \mathrm{~J}$ Watson, ${ }^{5} \mathrm{R}$ Pearson, ${ }^{4} \mathrm{I}$ Holbrook, ${ }^{6} \mathrm{~N}$ Durham, ${ }^{6} \mathrm{M}$ Pye. ${ }^{1}$ Division of Biostatistics, University of Leeds, Leeds, UK;
${ }^{2}$ Department of Respiratory Medicine, York Hospitals NHS Foundation Trust, York, UK; ${ }^{3}$ Department of Echocardiography, York Hospitals NHS Foundation Trust, York, UK; ${ }^{4}$ Department of Biochemistry, York Hospitals NHS Foundation Trust, York, UK; ${ }^{5}$ Leeds Teaching Hospitals, Leeds, UK; ${ }^{6}$ Department of Cardiology, York Hospitals NHS Foundation Trust, York, UK

Background Brain natriuretic peptides have been shown to be reliable indicators of left ventricular failure and markers of risk in cardiac disease. However, patients with chronic obstructive pulmonary disease (COPD) are also known to have elevated concentrations of brain natriuretic peptides in the absence of overt cardiac disease, likely due to right ventricular strain. This has been shown to have prognostic value and has a potential role in the management of the condition; for example, it has been suggested that it could be used to guide the initiation of non-invasive ventilation. The aim of this study was to identify clinical and echocardiographic determinants of the polypeptide N-terminal proBrain Natriuretic Peptide (NT pro-BNP) in patients with stable COPD.

Method Arterial blood gases, plasma NT pro-BNP and transthoracic echocardiographic parameters were studied in 140 patients with stable COPD attending a respiratory outpatient clinic.

Results Of the 140 patients, 65 (46\%) were male, $26(19 \%)$ received home oxygen therapy, 115 (82\%) were current smokers, 38 (27\%) were prescribed diuretics and $15(11 \%)$ had a left ventricular ejection fraction $<45 \%$. Patients with cor pulmonale $(n=6)$ were more likely to have left ventricular systolic dysfunction $(p<0.001)$, reduced tricuspid annular plane systolic excursion $(p=0.017)$ and higher pulmonary artery systolic pressures $(p=0.01)$. The median (IOR) NT pro-BNP concentration was 16.2 (25.4) pmol/l. Concentrations were significantly higher in those with a dilated left atrium, aortic stenosis, left ventricular systolic dysfunction, right ventricular impairment, atrial fibrillation and those prescribed diuretics and ACE inhibitors. Significant predictors of NT pro-BNP were a dilated left atrium, aortic stenosis and left ventricular systolic dysfunction. NT Pro-BNP was an excellent discriminator of RV impairment (C statistic $=0.90$ )

Conclusions NT pro-BNP readily identifies patients with stable COPD who have right ventricular dysfunction. However, several other clinical variables also associated with increased NT pro-BNP concentrations are prevalent in this population. This is likely to confound clinical decision making.

\section{CHF PATIENTS ARE VITAMIN D DEFICIENT AND HYPERPARATHYROID, WITH LEVELS OF EACH RELATED TO MARKERS OF SEVERITY}

doi:10.1136/heartjnl-2011-300198.106

${ }^{1} \mathrm{G}$ A Begg, ${ }^{1} \mathrm{~L}$ Kearney, ${ }^{2} \mathrm{~A}$ C Wheatcroft, ${ }^{1} \mathrm{R}$ Byrom, ${ }^{1} \mathrm{~S}$ Barnes, ${ }^{1} \mathrm{~J}$ Gierula, ${ }^{2} \mathrm{~J}$ Barth, ${ }^{2} \mathrm{R}$ Cubbon, ${ }^{2} \mathrm{M}$ T Kearney, ${ }^{2} \mathrm{~K}$ K Witte. ${ }^{1}$ Leeds General Infirmary, Leeds, UK; ${ }^{2}$ University of Leeds, Leeds, UK

Background The vitamin D-parathyroid (PTH) axis is increasingly recognised as potentially being involved with many of the features of the syndrome of CHF. We wanted to explore the relationship between vitamin D and PTH levels in a group of CHF patients and relate these to markers of severity.

Methods We analysed serum 25(OH) vitamin D3 levels in 406 consecutive attendees of the Leeds Advanced Heart Failure clinic (310 men) and correlated these to clinical markers of severity.

Results Mean age (SE) was 69 (3) years, mean left ventricular ejection fraction (LVEF) 31 (2)\%, mean serum creatinine $117 \mu \mathrm{mol} / 1$ (2.4), median vitamin D levels (IOR) $30(20-43) \mathrm{nmol} / \mathrm{l}$ (normal for skeletal health $>75 \mathrm{nmol} / \mathrm{l}$ ) and median parathyroid levels 8.8 $(6.2-13.5) \mathrm{pmol} / \mathrm{l}$ (normal $<6.5 \mathrm{pmol} / \mathrm{l}$ ). Aetiology was ischaemic 